\title{
Analytical investigation of high velocity impact on hybrid unidirectional/woven composite panels
}

\author{
GH. Liaghat ${ }^{1,2}$, H. Shanazari ${ }^{1}$, H. Hadavinia ${ }^{2}$, A. Aboutorabi ${ }^{2}$ \\ ${ }^{1}$ Department of Mechanical Engineering, Tarbiat Modares University, P.O. Box 14115/143, Tehran, Iran \\ ${ }^{2}$ Material Research Centre, SEC Faculty, Kingston University, London, SW15 3DW, UK
}

\begin{abstract}
In addition to fiber properties, the fabric structure plays an important role in determining ballistic performance of composite body armor textile. Textile structures used in ballistic protection are woven fabrics, unidirectional fabric structures and nonwoven fabrics. In this paper an analytical model based on wave propagation and energy balance between the projectile and the target is developed to analyze hybrid fabric panels for ballistic protection. The hybrid panel consists of two types of structure, woven fabrics as the front layers and unidirectional (UD) material as the rear layers. The model considers different cross sections of surface of the target in the woven and unidirectional fabric of the hybrid panel. Also the model takes into account possible shear failure by using shear strength together with maximum tensile strain as the failure criteria. Reflections of deformation waves at interface between the layers and also the crimp of the yarn are modeled in the woven part of the hybrid panel. The results show that the greater efficiency of woven fibers in front (more shear resistance) and unidirectional yarns in the rear (more tensile resistance), lead to better ballistic performance. Also modeling the yarn crimp results in more trauma in the backface of the panel producing data closer to the experimental results. It was found that there is an optimum ratio of woven to UD materials in the hybrid ballistic panel.
\end{abstract}

Keyword: hybrid composite, high velocity, impact, analytical model

\section{Introduction}

Nowadays, hybrid panels with high strength yarns such as ultra-high molecular weight polyethylene (UHMWPE) are widely used for the ballistic protection because of their light weight and high performance against impact [1]. There are two basic types of materials used in soft armor construction, aramid (woven) and high performance polyethylene (UD) fibers. Investigation on the ballistic protection is focusing more on the development of a hybrid design and its architecture (a combination of woven and UD lay-up). In woven fabrics, crimp is formed due to the interlacing of warp and weft yarns. The impact affecting to the yarn during ballistic impact applies a tension to the yarn through its axes. When a projectile impacts a fabric plane, it deflects towards the backface of the panel. This causes the yarns to move towards the back of the fabric panel more and creates a deeper trauma. In fact due to the crossover points in woven composites, a greater extension of the ballistic wave is reflected back, rendering the ballistic impact to a smaller composite area and reducing the rate at which energy can be dissipated. To improve this disadvantage, a non-crimp fabric is made using unidirectional (UD) plies in warp and weft direction. Thus, the stress wave propagated on the fabric plane is more than transmitted to the back of the fabric layers. This forms a smaller depth of the backface and the damage size. Park et al. [2] found that by combining or hybridizing unidirectional/woven fabrics, the materials leads to a better ballistic performance, especially by increasing the energy absorption capability. In woven fabric 
construction weft yarns will break before warp yarns during ballistic impact event [3]. Hence, a basic fundamental study is required for each individual architecture before employing them in hybrid model. One important step to design the hybrid panels is the combination of woven fabrics layers with unidirectional (UD) fiber reinforced laminates.

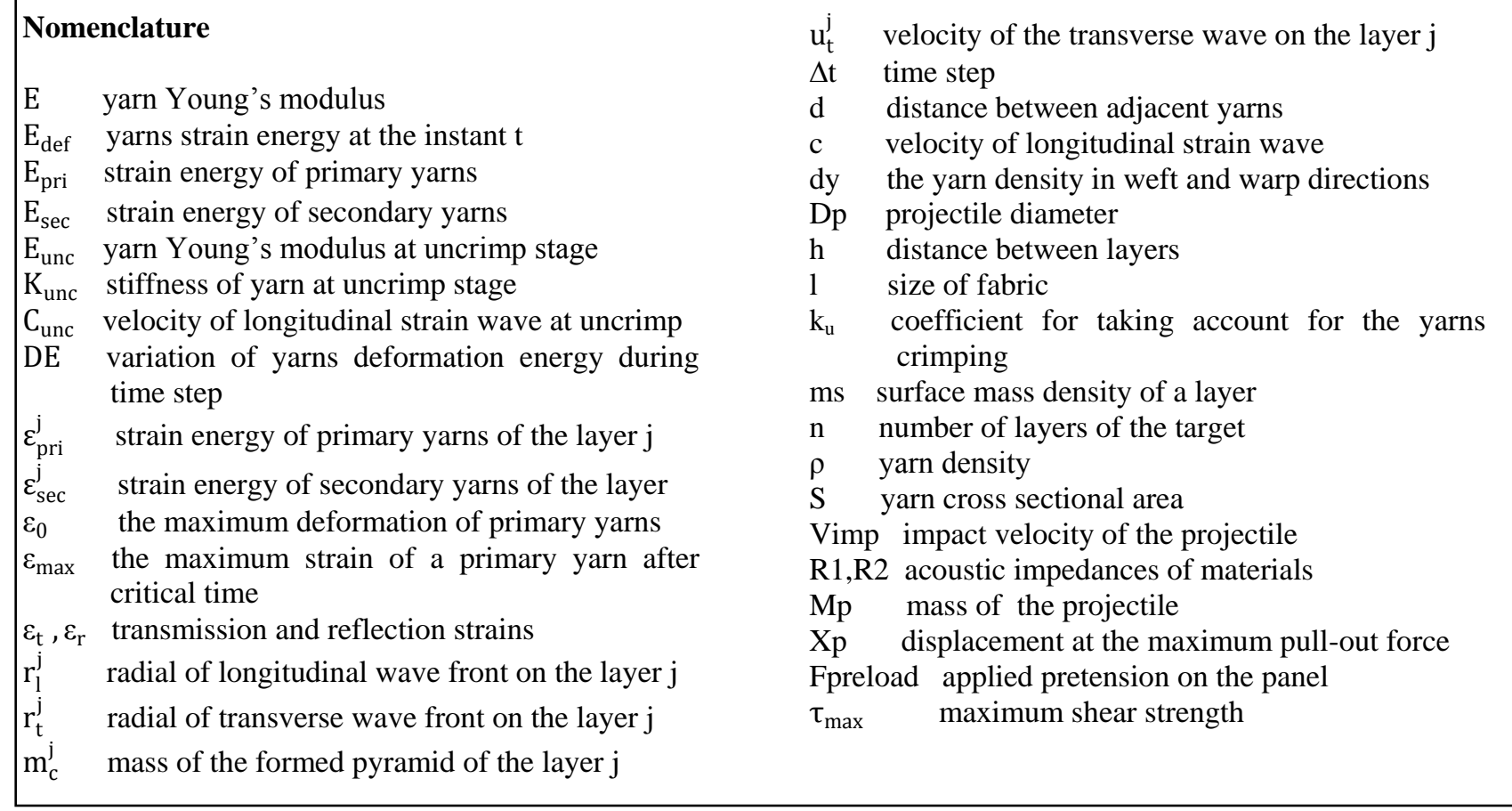

In woven fabrics, due to the existence of intersection point among the fibers, filaments are not stretched along their axis until the yarns are fully uncrimped. This decreases the velocity of the longitudinal strain wave and affects how the yarn performance is exhibited. In fact because of the existence of non-crimped yarn, the longitudinal strain wave travels faster in UD fabrics than in woven fabrics. This leads to participation of more fabric material in energy absorption process. Hybrid panels formed by both UD fabrics and woven fabrics show better performance than single material panels. Experimental investigation carried out by Thomas [3] showed that single material aramid panels made more depth of backface signature than hybrid panels and thus these panels demonstrate a higher ballistic resistance than single UD material or woven fabric panels. Karahan et al. [4] found that about $13.9 \%$ less energy was transmitted to the backing material in a hybrid panel than in a single UD material panel.

The penetration and perforation of composite targets by projectiles involve complex processes which have been studied analytically during the last few decades [5,6]. Many parameters affect on ballistic performance of hybrid composite targets such as materials, structures of composite, dimensions, boundary conditions and etc [7,8,9]. Smith et al. [10] studied the behaviour of an infinite long yarn under transverse impact. They showed that the impact creates longitudinal and transversal waves on a single yarn which propagate down the yarn. The shape of the transverse wave is like an inverted $V$ shape.

Bazle Z (Gama) Haque and John W Gillespie Jr. [11] developed a new penetration equation base on new definition of the projectile instantaneous velocity for ballistic limit analysis. 
With this new definition, the new penetration equation is derived satisfying both the conservation of momentum and energy principles simultaneously.

Roylance [12] studied the effects of yarn linear viscoelasticity using a finite-element simulation and obtained many behavioral features seen in experiments. Naik et al. [13] presented an analytical model to predict ballistic limit velocity, residual velocity and the energy absorbtion of two-dimensional woven fabric composites. This model is based on wave theory, dynamic mechanical and fracture properties of the textile composite.

Mamivand and Liaghat [14] developed an analytical model of two-dimensional woven fabric based on momentum and wave theory. The model allowed variation of distance between layers in order to study its effect on the ballistic resistance of the composite target.

Ha-Minh et al. [15] developed an analytical model based on both energy conservation and strain wave theory for $2 \mathrm{D}$ plain-weave fabrics subjected to ballistic impact. Reflections of strain waves are considered in this model. The effects of the dimensions of the panel and the distance between layers on its ballistic performance are also studied.

Mohan and Velu [16] developed a modified analytical model, based on Naik [13] model, to predict the impact behavior of unidirectional cross ply laminates. This model can predict the energy absorbed by different damage and energy absorbing mechanisms. Chen et al. [17] studied the behavior of hybrid fabric panels subjected to transverse impact. The finite element method was used to predict the response of different layers of a hybrid fabric model upon impact. Chen et al. [18] also developed a modified analytical model based on the momentum theory, taking into account the strain gradient between the panel layers and its effects on the tensile strain at the edge of the projectile.

Most of the studies presented earlier developed an analytical model for woven fabric panels. So an attempt is made to develop an analytical model to predict the impact behaviour of hybrid panel. This paper presents an analytical model based on wave propagation and energy balance to analyze hybrid fabric panels for ballistic protection. The architecture of this hybrid panel consists of two types of structure, woven fabrics as the front layers and unidirectional (UD) fabrics as the rear layers. The UD fabric used in this investigation are laminated at $0^{\circ}$ and $90^{\circ}$ alternately.

\section{Analytical model}

During impact, behavior of hybrid panel is different in woven fabric and unidirectional part. At woven fabric, after the impact of projectile to the hybrid panel, stress wave propagation and properties are the same in weft and warp directions while in the unidirectional part of the hybrid panel, properties along the yarn and in the direction normal to the yarns is different. In this section, an analytical model is developed to predict the evolution of projectile velocity, the deformation and absorption energies of these two parts (woven and unidirectional fabric) of a hybrid panel during impact. This analytical model takes into account possible shear failure by using shear strength together with maximum tensile strain as the failure criteria. The crimp of the yarn in the woven part of the hybrid panel and also reflections of deformation waves at interface between the layers are modeled. This model is based on the principle of energy conservation. As assumed by Ha-Minh et al. [15], during the impact event the projectile is perfectly rigid, strain of secondary yarns is distributed linearly from the edge of primary yarns to the transverse strain front and all primary yarns in a layer have the same 
deformation at any time. With these assumptions three main mechanisms of energy absorption are considered in this analytical model: kinetic energy of formed pyramid, failure and deformation of primary yarns and deformation of secondary yarns. Figure. 1 shows the primary and secondary yarns in a hybrid panel.

In this model, analysis is based on layer by layer system. This means that with considering a time interval, $\Delta \mathrm{t}$, depth of penetration of projectile is calculated depending on whether the next layer will be activated or not. If the next layer has been activated, all parameter such as velocity of projectile, mass of pyramid, amount of absorbed strain energy and kinetic energy can be calculated.

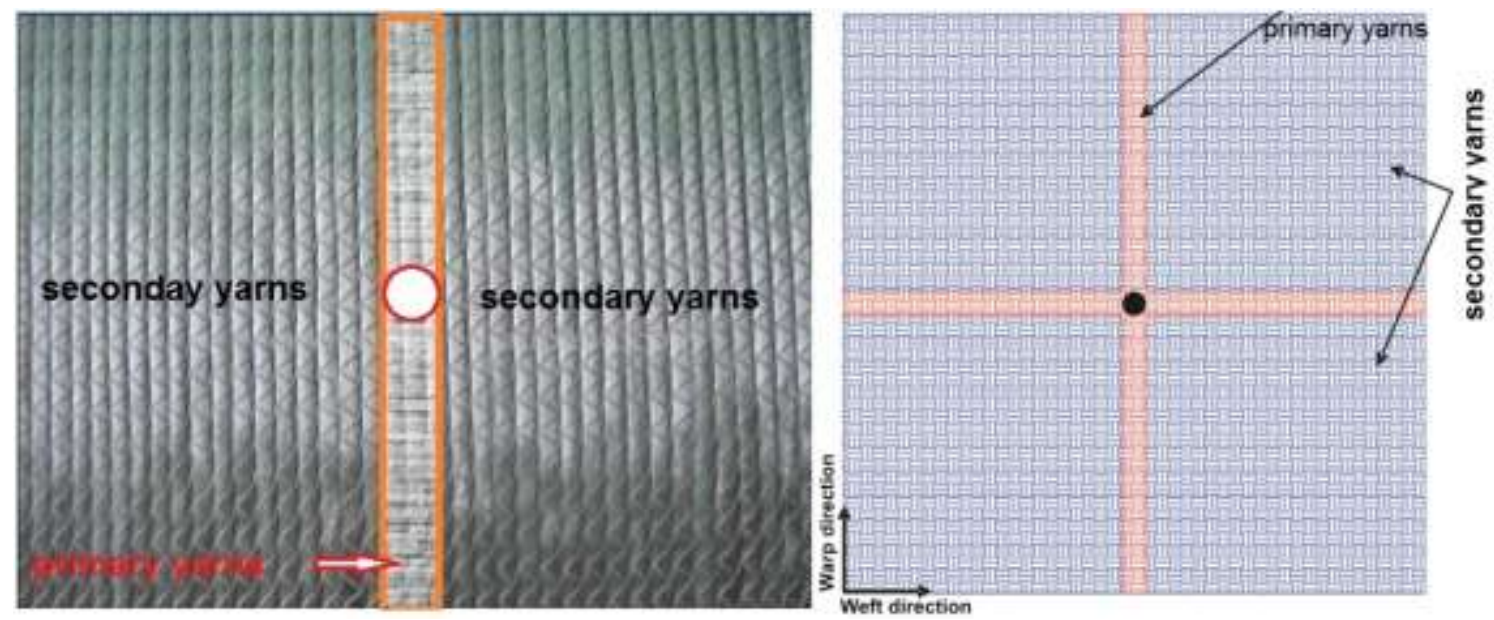

Fig.1.Primary and secondary yarns in unidirectional (left) and woven fabric (right).

\subsection{Absorbed energies}

According to Smith et al. [10] when the projectile is in contact with the yarn, ahead of the elastic wave front the strain is zero, and behind it, the strain is constant and can be calculated from:

$\sqrt{\varepsilon[2 \sqrt{\varepsilon(\varepsilon+1)}-\varepsilon]}=\frac{V_{\text {impact }}}{c}$

where $c=\sqrt{\frac{E}{\rho}}$ is the velocity of the longitudinal wave and $E$ is the yarn Young's modulus and $\rho$ is the yarn density. As the projectile impacts onto the woven fabric composite, a wave of deformation $\left(\varepsilon_{0}\right)$ starts to propagate on primary yarns. Because of interlacing of warp and weft yarn, this wave reaches the intersection point and reflected there(Figure.2). With these assumptions, the critical time, $\mathrm{t}_{\text {critical }}$ can be introduced as follows:

$\mathrm{t}_{\text {critical }}=\frac{2 \mathrm{~d}}{\mathrm{c}}$

where $d$ is the distance between adjacent yarns, $c=\sqrt{\frac{E}{\rho}}$ is the velocity of longitudinal strain wave on a yarn. When strain wave, $\varepsilon_{0}$ reaches the intersection point (two different materials), transmission strain wave $\varepsilon_{\mathrm{t}}$ and reflection strain wave $\varepsilon_{\mathrm{r}}$ are created which can be determined by the following equations:

$\varepsilon_{\mathrm{t}}=\frac{2 \mathrm{R}_{1}}{\mathrm{R}_{1}+\mathrm{R}_{2}} \varepsilon_{0}$ and $\varepsilon_{\mathrm{r}}=\frac{\mathrm{R}_{2}-\mathrm{R}_{1}}{\mathrm{R}_{1}+\mathrm{R}_{2}} \varepsilon_{0}$ 
where $R_{1}$ and $R_{2}$ are acoustic impedances. It can be noted that the mass density of yarns is doubled at the intersection points [16]. So, if the acoustic impedance of yarn, $R_{1}=\sqrt{\rho E}$, then it can be written $R_{2}=\sqrt{2 \rho E}$. Also because of the symmetry of this impact system, the reflection coefficient $r=\frac{R_{2}-R_{1}}{R_{1}+R_{2}}$ is doubled (2r) at the impact point. Reflection of the strain wave will be different at the unidirectional fabric of the hybrid panel, because of the non woven structure.

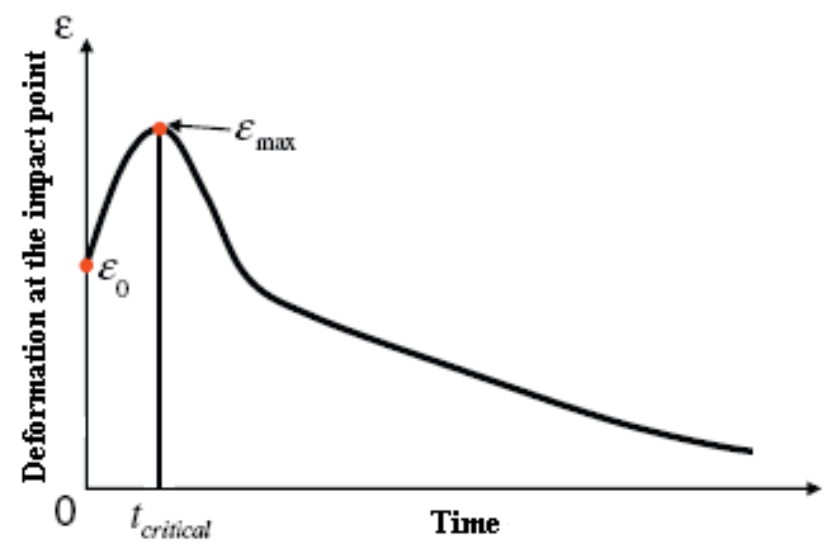

Fig2. Local growth of the strain at the impact point due to waves reflections [15].

In other word the reflection coefficient is not doubled at the impact point:

$r=2 \times \frac{R_{2}-R_{1}}{R_{1}+R_{2}} \quad$ for woven structure

$\mathrm{r}=\frac{\mathrm{R}_{2}-\mathrm{R}_{1}}{\mathrm{R}_{1}+\mathrm{R}_{2}} \quad$ for UD structure

The local strain at the point of impact, $\varepsilon_{\max }$ is sharply increased at the time, $t_{\text {critical }}$, after impact and can be determined by:

$\varepsilon_{\text {max }}=\varepsilon_{0}+\mathrm{k}_{\varepsilon} . \varepsilon_{\mathrm{r}}$

where $\varepsilon_{0}$ is the deformation of primary yarns immediately after impact, $\mathrm{k}_{\varepsilon}$ is the coefficient to take into account the interference of strain waves. This coefficient can be taken equal to 2.0 for woven fabric [12] and 1.0 for unidirectional layers.

The energy and momentum conservation law can be used during impact. In the time interval $\Delta t$ the equation of energy conservation can be written as follows:

$\frac{1}{2}\left(M_{c}(t)+M_{p}\right) v(t)^{2}=\frac{1}{2}\left(M_{c}(t-\Delta t)+M_{p}\right) v(t-\Delta t)^{2}-D E(t)$

where $\mathrm{M}_{\mathrm{c}}(\mathrm{t})$ and $\mathrm{v}(\mathrm{t})$ are the mass of the fabric cone and the velocity of the projectile respectively, $\mathrm{DE}(\mathrm{t})$ is the variation of deformation energy during a time interval. The mass of the formed pyramid of the layer $\mathrm{j}$ at the instant $\mathrm{t}$ can be calculated as follow:

$\mathrm{M}_{\mathrm{c}}(\mathrm{t})=\sum_{\mathrm{j}=1}^{\mathrm{n}} \mathrm{m}_{\mathrm{s}} \cdot \pi \cdot \mathrm{r}_{\mathrm{t}}^{\mathrm{j}}(\mathrm{t})^{2}$

Where $r_{t}^{j}(t)$ is the radius of transverse wave front on the layer $j$ at the instant $t$ and $m_{s}$ is the surface density of a layer. The radius of transverse wave front on the layer $\mathrm{j}$ is calculated in a time step as follows:

$\left.r_{\mathrm{t}}^{\mathrm{j}}(\mathrm{t})=r_{\mathrm{t}}^{\mathrm{j}}(\mathrm{t}-\Delta \mathrm{t})+\mathrm{u}_{\mathrm{t}}^{\mathrm{j}}(\mathrm{t}) \cdot \Delta \mathrm{t}\right)$ 
where $u_{t}^{j}(t)$ is the velocity of the transverse wave on the layer $j$ at the instant $t$ and determined by:

$$
u_{t}^{j}(t)=c \cdot\left[\sqrt{\varepsilon_{p r i}^{j}(t)\left(\varepsilon_{p r i}^{j}(t)+1\right)}-\varepsilon_{p r i}^{j}(t)\right]
$$

The strain energy of primary yarns of the layer $\mathrm{j}$ at the instant $\mathrm{t}$, can be determined by the following relationship:

$$
e_{p r i}^{j}(t)=E \cdot \varepsilon_{p r i}^{j}(t)^{2} \cdot V
$$

where:

$V=2 D_{p} \cdot d_{y} \cdot S \cdot r_{l}^{j}(t) \quad$ for woven structure

$V=D_{p} \cdot d_{y} \cdot S \cdot r_{l}^{j}(t) \quad$ for UD structure

and $\varepsilon_{\text {pri }}^{\mathrm{j}}(\mathrm{t})$ is the deformation of primary yarns of the layer $\mathrm{j}$ at the instant $\mathrm{t}, V$ is the volume of the deformed primary yarn of the layer $\mathrm{j}, \mathrm{S}$ is the area of cross section of a yarn and $\mathrm{d}_{\mathrm{y}}$ is density of yarns.

\subsubsection{Deformation energy of secondary yarns in woven fabric}

It can be noted that the deformation zone of secondary yarns on the layer $\mathrm{j}$ limits between two radii: $D_{p} / \sqrt{2}$ and $r_{t}^{j}(t)$. Therefore, the deformation energy of the secondary yarns of the layer $\mathrm{j}$ at the instant $\mathrm{t}$ is determined by the following relationship:

$\mathrm{E}_{\mathrm{sec}}^{\mathrm{j}}(\mathrm{t})=\int_{D_{\mathrm{p}} / \sqrt{2}}^{\mathrm{r}_{\mathrm{t}}^{\mathrm{j}}(\mathrm{t})}\left(\int_{0}^{\varepsilon_{\text {sec }}^{\mathrm{j}}(\mathrm{t})} \sigma_{\text {sec }}^{\mathrm{j}}\left(\varepsilon_{\sec }^{\mathrm{j}}\right) \mathrm{d} \varepsilon_{\text {sec }}^{\mathrm{j}}\right) \cdot \mathrm{dV}$

Which $d V$ is the volume increment of the secondary yarns zone between $\mathrm{r}$ and $(\mathrm{r}+\mathrm{dr})$ shown in Figure. 3 and determined by the following formulation:

$d V=h \cdot\left\{2 \pi r-8 r \sin ^{-1}\left(\frac{D_{p}}{2 r}\right)\right\} \cdot d r$

Therefore:

$\left.E_{\text {sec }}^{j}(t)=\int_{D_{p / \sqrt{2}}^{r_{t}^{j}}(t)}^{r_{0}^{\varepsilon_{\text {sec }}^{j}(t)}} \sigma_{\text {sec }}^{j}\left(\varepsilon_{\text {sec }}^{j}\right) d \varepsilon_{\text {sec }}^{j}\right) \cdot h \cdot\left\{2 \pi r-8 r \sin ^{-1}\left(\frac{D_{p}}{2 r}\right)\right\} d r$

\subsubsection{Deformation energy of secondary yarns in unidirectional fabric}

An elliptical cone is formed on the back face of the panel during impact. Due to orthotropic nature of the unidirectional fabric, the shape of the wave front would be elliptical. The surface area of the elliptical cone can be determined based on transverse wave propagation. During impact event the transverse wave moves from $x_{t 1}$ to $x_{t 2}$ in $x$ direction and from $y_{t 1}$ to $\mathrm{y}_{\mathrm{t} 2}$ in $\mathrm{y}$ direction. The distance $\mathrm{x}_{\mathrm{t} 2}$ and $\mathrm{y}_{\mathrm{t} 2}$ are determined based on additional transverse wave propagation which has been taken place during that time step.

As mentioned before, because of the orthotropic nature of the panel, the strain variation is different in directions $x$ and y. As shown in Fig. 3 the strain variation is assumed to be linear in both directions OA and OB. The strain at the boundary of cone is assumed to be zero to simplify the model. Based on the assumption, boundary conditions can be presented by following equations:

$\mathrm{x}=0, \mathrm{y}=0: \varepsilon_{\text {secx }}^{j}(t)=\varepsilon_{\text {secy }}^{j}(t)=\varepsilon_{\text {pri }}^{j}(t)$

$\mathrm{x}=\mathrm{x}_{\mathrm{t}}: \varepsilon_{\text {secx }}^{j}(t)=0$ 
$\mathrm{y}=\mathrm{y}_{\mathrm{t}}: \varepsilon_{\text {secy }}^{j}(t)=0$

From these boundary conditions, the strain can be formulated in $\mathrm{x}$ and $\mathrm{y}$ direction as:

$\varepsilon_{s e c x}^{j}(t)=\frac{\mathrm{x}_{\mathrm{t}}-\mathrm{x}}{\mathrm{x}_{\mathrm{t}}} \varepsilon_{p r i}^{j}(t)$

$\varepsilon_{s e c y}^{j}(t)=\frac{\mathrm{y}_{\mathrm{t}}-\mathrm{y}}{\mathrm{y}_{\mathrm{t}}-\mathrm{r}_{\mathrm{p}}} \varepsilon_{p r i}^{j}(t)$

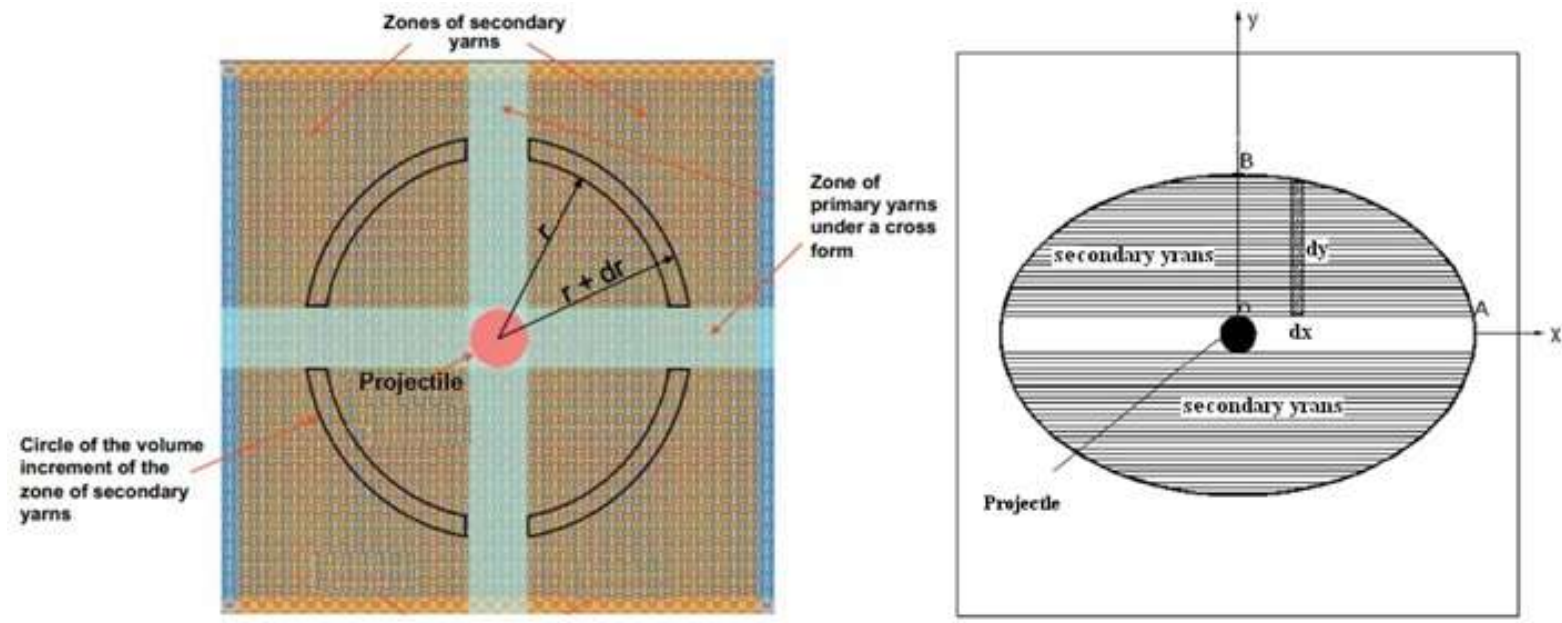

Fig.3 Modeling of deformation of secondary yarns in woven (left) and unidirectional (right) fabric.

The energy absorbed by deformation of secondary yarns can be obtained by:

$E_{s e c}^{j}(t)=\int_{0}^{\mathrm{x}_{\mathrm{ti}}} e_{s e c}^{j}(t) \cdot 4 \mathrm{~h}\left[\left(\mathrm{y}_{\mathrm{t}}-\mathrm{D}_{\mathrm{p}} / 2\right) \sqrt{\frac{\mathrm{x}_{\mathrm{t}}^{2}-\mathrm{x}^{2}}{\mathrm{x}_{\mathrm{t}}^{2}}}\right] \mathrm{dx}$

The variation of strain energy of yarns in the time interval $\Delta t$ can be formulated by the following equation:

$D E(t)=E_{d e f}(t)-E_{d e f}(t-\Delta t)$

where $E_{\text {def }}(t)$ and $E_{\text {def }}(t-\Delta t)$ are strain energies of yarns at the instant $t$ and $(t-\Delta t)$. Yarns strain energy $E_{\text {def }}(t)$ is the sum of the strain energy of primary yarns and strain energy of secondary yarns. The total strain energy of $n$ layers for primary and secondary yarns is calculated as following equation:

$E_{\text {def }}(\mathrm{t})=\mathrm{E}_{\text {pri }}(\mathrm{t})+\mathrm{E}_{\mathrm{sec}}(\mathrm{t})=\sum_{\mathrm{j}=1}^{\mathrm{n}} \mathrm{e}_{\mathrm{pri}}^{\mathrm{j}}(\mathrm{t})+\sum_{\mathrm{j}=1}^{\mathrm{n}} \mathrm{e}_{\text {sec }}^{\mathrm{j}}(\mathrm{t})$

\subsection{Failure criteria}

In the analytical model presented here, possible shear failure is considered by using shear strength together with the maximum tensile strain as the failure criteria. Lim et al. [19] found that aramid fibers fibrillate under high strain rate compression, and this means that the main failure mechanism of the transversely impacted fiber was related to fibrillation. Chen et al. [17] showed in their experiments that the failure tensile strength is low for the front layers and it turns to be higher for the rear fabric layers and the failure shear strength for the front layers is high and it becomes lower for the rear fabric layers. This means that the main reason of failure of fabric layers at the front of the panel is due to shear stresses. 
Cheng et al. [20] also investigated that the main reason of failure of aramid fiber was fibrillation. It is assumed that fibrillation of fibers is regarded as fiber failure in this analytical model. Thus, two failure criteria applied for the model in the study. On the other hand:

$\varepsilon_{\text {pri }}^{\mathrm{j}}(\mathrm{t})>\varepsilon_{\max }$ and $\sigma_{\text {pri }}^{\mathrm{j}}(\mathrm{t})>2 \tau_{\max }$

where $\varepsilon_{\max }$ is tensile strain failure of fiber, $\sigma_{\text {pri }}^{\mathrm{j}}(\mathrm{t})$ is the compressive stresses in primary yarns at instant $\mathrm{t}$ and $\tau_{\max }$ is maximum shear strength of fiber.

\subsection{Modeling of crimp in the woven fabric part}

In woven fabrics crimps are formed because of the interlacing in warp and weft yarns. Crimp of fiber yarns decreases the in-plane properties leading in serious failures such as fiber kinking that affects the ballistic performance of panel. Because the velocity of strain wave is different in uncrimping stage thus it is necessary to calculate the strain at this stage. To do this the yarn pull out test and affecting parameters on this phenomenon need to be studied. Yarn pull-out can be an important mechanism of energy absorption during the ballistic impact [21]. In the yarn pull out test one or two lateral edges will be fixed and one or more threads are pulled out. On this basis, variation of pull out force versus displacement can be plotted. As shown in Fig.4 'uncrimping' stage is the beginning of the force-displacement curve and 'yarn translation', corresponding to the portion past the peak load point. The uncrimping process is irreversible meaning that the yarn will not return to the original crimped configuration. The yarn uncrimping and yarn translation process dissipate some energy defined as the area under the force-displacement curve.

$\mathrm{P}_{\max }$ the maximum force required pulling out this yarn and $\mathrm{X}_{\mathrm{p}}$ the displacement at the maximum force depend on various parameters and increase linearly with increasing of yarn length. As the number of neighboring yarns pulled during a test increases, the peak load and displacement at peak load point increase too. Kirkwood et al. [22] presented the following equation for pull out $\mathrm{n}$ yarn simultaneously:

$\mathrm{P}_{\max }=\frac{2 \mathrm{n}-1}{\mathrm{n}} \mathrm{P}_{\max }^{\mathrm{n}=1}$

If $K_{u n c}$ is the stiffness of a yarn during uncrimping phenomena [23], then:

$K_{\text {unc }}=\frac{P_{\max }}{X_{p}}$

Now with assumption of linear relation between stress-strain, it can be concluded:

$\mathrm{E}_{\text {unc }}=\frac{\sigma}{\varepsilon}=\frac{\mathrm{P}_{\max } / \mathrm{S}}{\mathrm{X}_{\mathrm{p}} / \mathrm{L}}=\frac{\mathrm{K}_{\mathrm{unc}} \cdot \mathrm{L}}{\mathrm{S}}$

Where $\mathrm{E}_{\mathrm{unc}}$ is the yarn longitudinal Young's modulus during uncrimping stage, $\mathrm{S}$ is crosssection and $\mathrm{L}$ is length of the yarn.

Now the velocity of strain wave created in the yarn during uncrimping after impact can be determined as following:

$C_{\text {unc }}=\sqrt{\frac{E_{\text {unc }}}{2 \rho}}=\sqrt{\frac{\mathrm{K}_{\mathrm{unc}} \cdot \mathrm{L}}{2 \rho \mathrm{S}}}$ 
Therefore, $\mathrm{C}_{\mathrm{unc}}$ can be used instead of $\mathrm{C}$ to calculate the velocity of the transverse wave. In the analytical model developed by Ha-Minh [15] the following equation used to calculate the velocity of transverse wave during impact:

$\mathrm{u}_{\mathrm{t}}^{\mathrm{j}}(\mathrm{t})=\mathrm{k}_{\mathrm{u}} \cdot \mathrm{C} \cdot\left[\sqrt{\varepsilon_{\text {pri }}^{\mathrm{j}}(\mathrm{t})\left(\varepsilon_{\text {pri }}^{\mathrm{j}}(\mathrm{t})+1\right)}-\varepsilon_{\text {pri }}^{\mathrm{j}}(\mathrm{t})\right]$

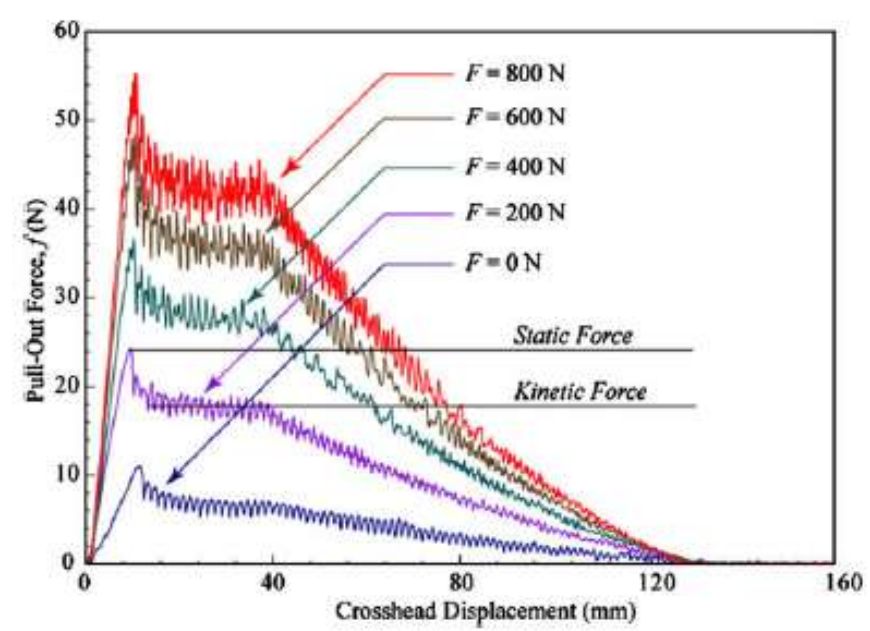

Fig. 4. Yarn pull-out force versus crosshead displacement for varying magnitudes of the applied pre-load [24] .

It is noted that the coefficient $\mathrm{k}_{\mathrm{u}}$ which takes into account the effect of the weaving (such as crimping) on the velocity of the transverse wave can vary as a function of time. Therefore combination of equations 25 and 26 result in:

$u_{t}^{j}(t)=k_{u} \cdot C \cdot f(\varepsilon)=k_{u} \cdot \sqrt{\frac{E_{u n c}}{2 \rho}} \cdot\left[\sqrt{\varepsilon_{p r i}^{j}(t)\left(\varepsilon_{p r i}^{j}(t)+1\right)}-\varepsilon_{p r i}^{j}(t)\right]$

Thus:

$\mathrm{k}_{\mathrm{u}}=\sqrt{\frac{\mathrm{K}_{\mathrm{unc} \cdot \mathrm{L}}}{\mathrm{E} \cdot \mathrm{S}}}$

Investigations show that the lateral force (preload) in the pull out test, affects on pull-out force. Kirkwood [22] reported by increasing the transverse fabric tension, the yarn pullout energy increases. In fact increasing the tension on the transverse yarns increases normal forces between yarns, resulting in more frictional resistance to yarn pull-out and also a more tortuous path during yarn translation in the pull-out phenomena.

In force-displacement curve (Fig.4), the displacement at peak load is approximately independent of transverse tension since the length scale of the pull-out curve is primarily determined by the length of the fabric specimen. Rao et al. [24] also investigated that linear relationship could be employed to predict pull-out force in terms of the applied preload. The linear relationship equation can be written as:

$\mathrm{P}_{\text {max }}=\mathrm{m}_{1} \cdot \mathrm{F}_{\text {preload }}+\mathrm{m}_{2}$

where $\mathrm{P}_{\max }$ is the average yarn pull-out force, $\mathrm{F}_{\text {preload }}$ indicates the applied preload and $m_{1}$ and $m_{2}$ are the constant coefficient of the linear relationship. According to equations (14-17), it can be concluded that coefficient $\mathrm{k}_{\mathrm{u}}$ is related to $\mathrm{F}_{\text {preload }}, \mathrm{K}_{\mathrm{unc}}$. In other word:

$\mathrm{k}_{\mathrm{u}}=f\left(\mathrm{~F}_{\text {preload }}, \mathrm{K}_{\mathrm{unc}}\right)$ 


\section{Results and discussion}

As discussed before, three mechanisms of energy absorption are considered in our model, i.e. kinetic energy of pyramid forming, deformation of primary yarn and deformation and strain energy of secondary yarn. These energy absorption mechanisms are applied on woven fabric and unidirectional plies of hybrid panel.

According to equations in this paper all parameters of penetration process such as absorbed energies, velocity of projectile and height and width of the pyramid on the backface of the panel at each time interval can be calculated. It is vital to emphasis that all parameters at any time ( $\mathrm{t}$ ) can be determined from the projectile velocity at the time ( $\mathrm{t}-\Delta \mathrm{t})$. Thus, the time interval must be chosen small enough to obtain accurate results, but too small time step will increase the computational time. Therefore, there is an optimum time step for any impact velocity. The software MATLAB is used to calculate the parameters and to obtain the results. The material used in woven section is a Style $745 \mathrm{~S}$ plain-weave fabric of $30.5 \times 30.5 \mathrm{~cm}$. Material's properties were taken from Ref. [8]. The area density of a single layer is $448 \mathrm{~g} / \mathrm{m}^{2}$. The thickness of a single layer is $0.05 \mathrm{~cm}$. The whole target is 10 layers and a space of 0.05 $\mathrm{mm}$ is left between the layers. For the fabric longitudinal elastic modulus $96 \mathrm{GPa}$, and a failure strain of approximately 3\% is considered. The material used in unidirectional part is fabric made of Dyneema SB21 with areal density of $145 \mathrm{~g} / \mathrm{m}^{2}$. The projectile used in this model is fragment simulating projectile (FSP) type with mass of $2.82 \mathrm{~g}$ and diameter of 7.62 $\mathrm{mm}$. Initially the panel with $100 \%$ woven fabric structure is analyzed and results are compared with hybrid panel consist of woven and unidirectional fabric.

\section{Woven fabric}

Absorbed energy by these three mechanisms is compared in Fig. 5. As shown in this figure the penetration of the projectile can be divided into three main phases. For an impact velocity of $375 \mathrm{~m} / \mathrm{s}$ in the first phase, $(\mathrm{t}<150 \mu \mathrm{s})$ the kinetic energy of the pyramid and the deformation energy of the primary yarns contribute primarily to deceleration of the projectile. In phase 2 $(150<\mathrm{t}<600 \mu \mathrm{s})$, the development of the pyramid deformation is the main mechanism for the energy absorption. In the last section $(t>600 \mu \mathrm{s})$, the deformation energy of yarns is the main mechanism of energy dissipation. At the time $t=500 \mu \mathrm{s}$ dimensions of the pyramid has reached to the edge of the panel. Therefore, the kinetic energy of this pyramid decreases with the projectile velocity. On the other hand, the deformation energies of primary and secondary yarns are rising sharply. It can be noted that because of more secondary yarns than the primary ones, the secondary yarns contribute largely to the deformation energy of the fabric. As mentioned before, the crimp of the yarn in the woven fabric is modeled in this study. Fig. 6 shows energy absorption by pyramid forming of the panel in two cases: no crimp in fabric and crimp yarns with $\mathrm{K}_{\mathrm{unc}}=5000 \mathrm{~N} / \mathrm{m}$ [25]. As shown in this figure the energy absorbed by pyramid forming of the panel with no crimp is more than the case with Kunc=5000 N/m.

Fig. 7 shows depth of backface signature in two cases: no crimp in fabric and crimp yarns with $K_{u n c}=5000 \mathrm{~N} / \mathrm{m}$. As shown in this figure the backface of the panel in case of $K_{u n c}=5000$ $\mathrm{N} / \mathrm{m}$, is more than the case with no crimp which is closer to the experimental result. In fact in the case of woven fabric, as the yarns are interlaced together, the fabric structure permits relative movement of the yarns in the fabric. When struck by a projectile, primary yarns are 
stretched and pulled out. It is evident that the limitation of fiber movement in the UD fabrics leads to smaller depth of backface than the woven fabric. Therefore, majority of the impact stress is propagated on the fabric plane and less is transmitted to the back of the fabric layers. This forms a smaller height of pyramid.

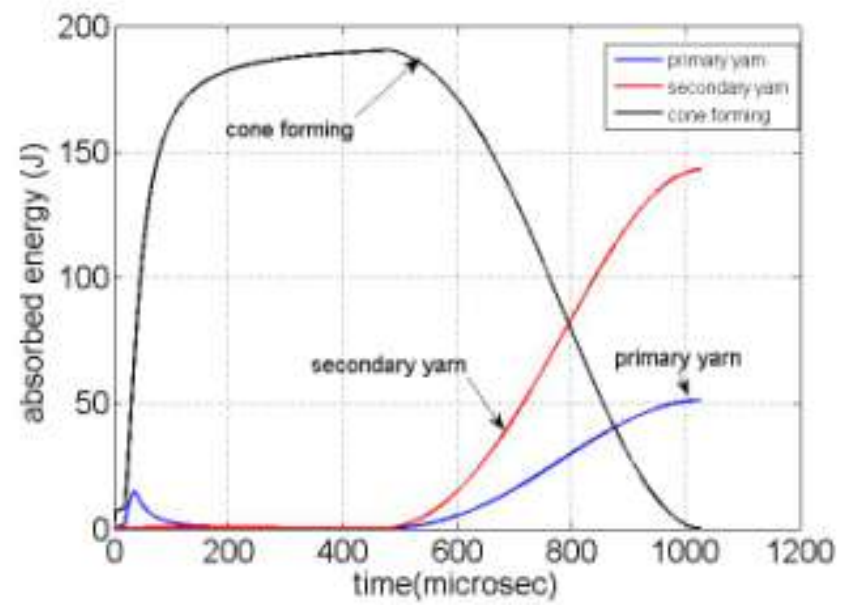

Fig.5. Comparison of absorbed energy in primary and secondary yarns and pyramid formed during impact.

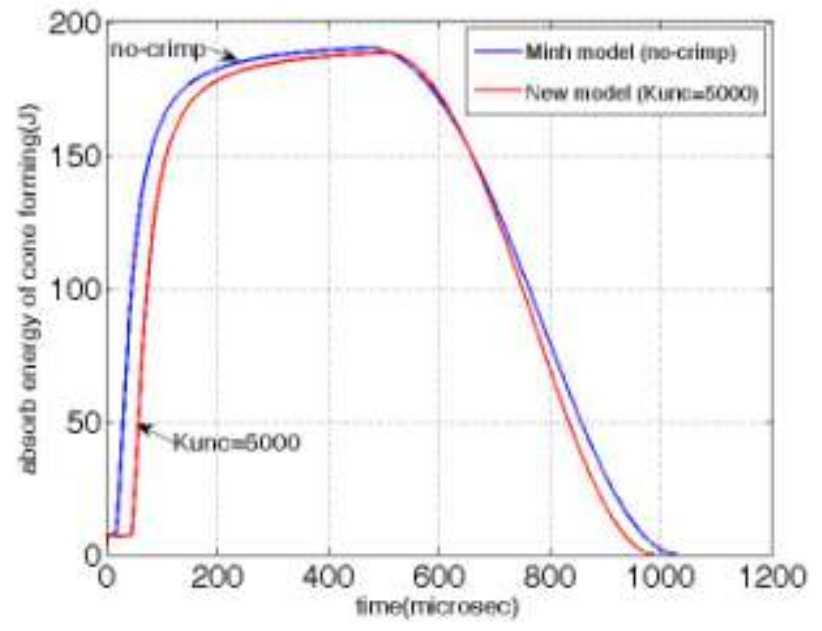

Fig.6. Comparison of absorbed energy in pyramid forming in the new model and Minh[12].

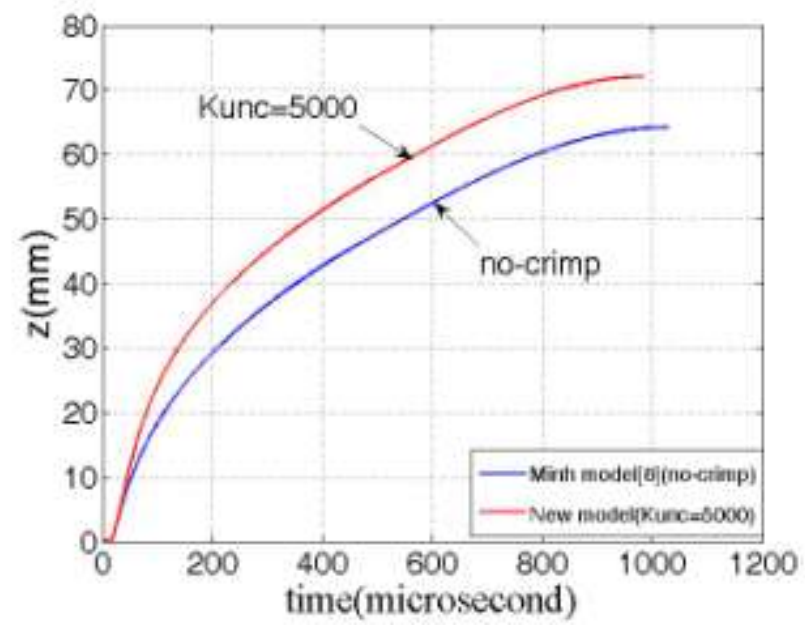

Fig. 7.Effect of crimp on the depth of backface of the panel in two cases, no crimp and crimp with stiffness Kunc=5000 N/m. 
On the other hand, it is evident that the in-plane fiber continuity and linearity of UD make the stress waves produced by high velocity projectiles propagating rapidly and effectively. As compared to UD, the existence of interlacing points between yarns in woven fabric restricts the projectile energy to be dissipated over a shorter time.

Hybrid panel

Experimental tests [17] showed that placing the woven fabric at the front face of the impact gave better ballistic protection for the panel than the reverse sequence. Therefore, the hybrid panel considered to analyze in this study consists of woven fabrics as the front layers and unidirectional (UD) material as the rear layers. The UD fabric used in this investigation are laminated at $0^{\circ}$ and $90^{\circ}$ alternately. On this basis five combination of panel were designed. In order to better compare the panel performance, areal densities of different panels were considered as similar as possible. The sequence of panel are given in Table 1.

Results of this analytical model including depth and width of backface of pyramid of the panel and ballistic limit are presented in Table 2 at impact velocity of $375 \mathrm{~m} / \mathrm{s}$.

For example two combinations of hybrid panel, A (100\% woven fabric) and E (100\% UD) given in Table 2 can be compared. It is found that in case A, the depth of backface (height of pyramid) is $30 \%$ more than in the case E. Also width of the formed pyramid at the backface in case A is about $19 \%$ less than in the case E. In fact width of the formed pyramid resulted in distribution of strain energy basis on moving ahead of transverse strain wave. As shown in Table 2 the ballistic limits of the panel in case A is lower than in case E.

Figs. 8 and 9 show the variation of the height and the width of the formed pyramid during the penetration process in the pure woven fabric panel (combination A) and pure unidirectional panel (combination E). The width of transverse deflection is found to be higher in the UD panel than in the woven fabric panel as shown in Fig. 9. This demonstrate a larger surface area of UD plies contributed to the energy dissipation and thus leads to higher ballistic performance in the pure UD panel than in the pure woven panel.

Table 1. Architecture of panels

\begin{tabular}{ccccc}
\hline Combination & $\begin{array}{c}\text { Number of } \\
\text { woven } \\
\text { layers }\end{array}$ & $\begin{array}{c}\text { Number of } \\
\text { UD } \\
\text { layers }\end{array}$ & $\begin{array}{c}\text { Areal } \\
\text { Density } \\
\mathrm{g} / \mathrm{m}^{2}\end{array}$ & Panel model \\
\hline A & 10 & 0 & 4480 & \\
B & 9 & 2 & 4510 & \\
C & 5 & 9 & 4400 & \\
D & 2 & 15 & 4490 & \\
& & & & \\
E & & & & \\
& & & & \\
\end{tabular}


It can be seen from Table. 2 that combination D, consist of 2 layers of woven fabric and 15 layers of UD layers exhibits the lowest value of the height of the pyramid which indicates its higher performance over other panels. It is found that, beyond this proportion, an increase in the proportion of woven fabrics in the panel leads to an increase in backface signature value. The combination A, consisting of 10 layers of woven fabric gives the worst performance.

Table 2. Comparison of height and width of the back face pyramid and ballistic limit velocity for lay-up shown in Table 1.

\begin{tabular}{|c|c|c|}
\hline Combination & $\begin{array}{c}\text { Height of } \\
\text { Pyramid } \\
\mathrm{z}(\mathrm{mm})\end{array}$ & $\begin{array}{c}\text { Width of } \\
\text { pyramid } \\
\mathrm{r}(\mathrm{mm})\end{array}$ \\
\hline A & 17.4 & 34 \\
\hline B & 16.3 & 36 \\
\hline C & 14 & 38.2 \\
\hline D & 11.6 & 42.1 \\
\hline E & 12.4 & 40.1 \\
\hline
\end{tabular}

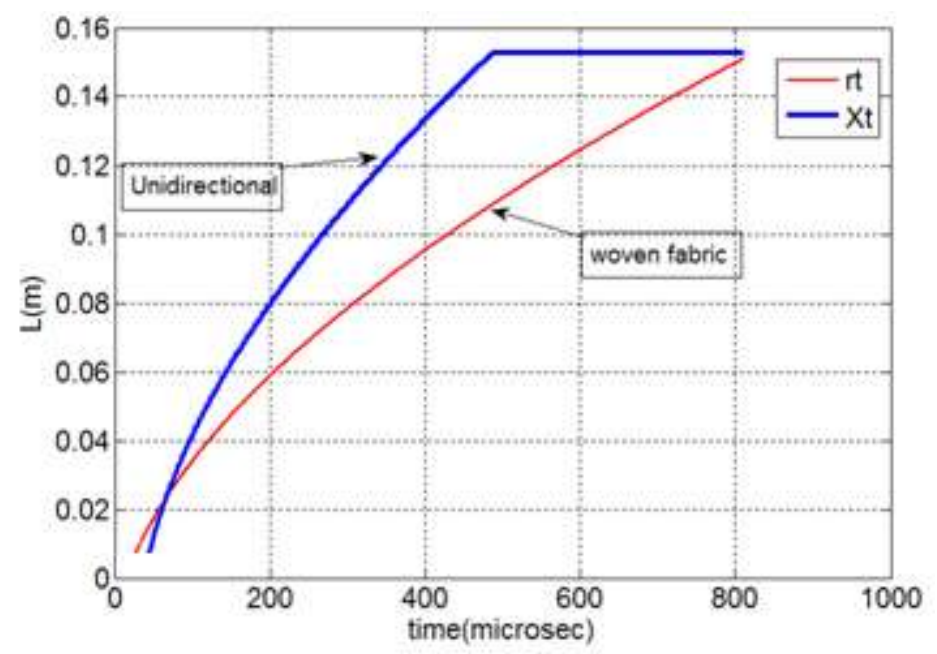

Fig. 8. Variation of width of the formed pyramid during the penetration process in pure woven and pure unidirectional panels (combinations $\mathrm{A}$ and $\mathrm{E}$ ). 


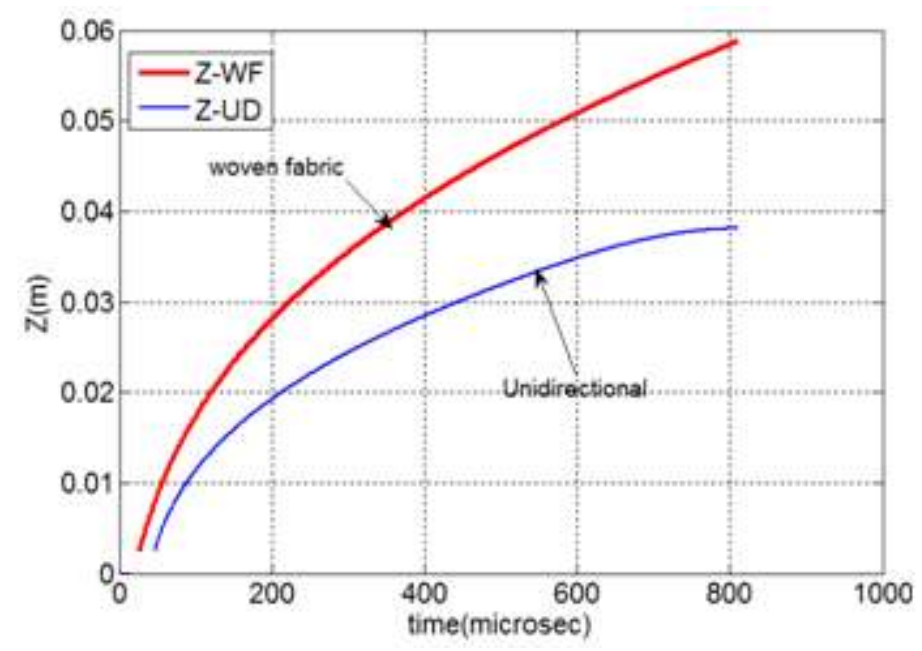

Fig.9. Variation of height of the formed pyramid during the penetration process in pure woven and pure unidirectional panels (combination $\mathrm{A}$ and $\mathrm{E}$ ).

For validation of the analytical model, experimental results by Chen et al. [17] have been used. Hybrid fabric samples used in this section contain a plain woven fabric made of Dyneema® ${ }^{\circledR}$ SK 75 and an unidirectional fabric made of Dyneema ${ }^{\circledR} \_S B 21$ with areal density of a single layer $240 \mathrm{gr} / \mathrm{m} 2$ and $145 \mathrm{gr} / \mathrm{m} 2$ respectively. Properties of the two fabrics are given in Chen et al. [17]. In this arrangement, the projectile is a $1 \mathrm{~g}$, cylindrical projectile whose length and diameter both measure $5.5 \mathrm{~mm}$. The average of the velocity of the projectile is about $450 \mathrm{~m} / \mathrm{s}$. Five configurations were designed as shown in Table 3 . In order to better compare the panel performance, the areal densities of different panels were kept as similar as possible.

Table 3. Type of panels

\begin{tabular}{lc}
\hline Composition & $\begin{array}{c}\text { Areal density } \\
(\mathbf{g r} / \mathbf{m} \mathbf{2})\end{array}$ \\
\hline 40 layers of UD fabric & 5800 \\
6 layers of woven + 30 layers of UD fabric & 5790 \\
12 layers of woven + 20 layers of UD fabric & 5780 \\
18 layers of woven + 10 layers of UD fabric & 5770 \\
24 layers of woven & 5760 \\
\hline
\end{tabular}

In Fig. 10 the depth of back face signature of the panel predicted by the analytical model compared with experimental results by Chen et al. [17]. As shown in Fig. 10 the combination of 6 layers of woven fabric and 30 layers of UD fabric shows the lowest value, which indicates its superior performance over other panels. Also this figure shows that results of the analytical model have acceptance consistency with experimental data. In this case the crimp of the woven part of the hybrid panel has not been considered.

In Fig. 11 the depth of the back face of the panel compared with experimental results by Chen Chen et al. [17]. In this configuration, the crimp of the woven part of the panel have been considered with Kunc=5000 N/m [25]. As shown in this figure, results of the analytical model are closer to the experimental data than before configuration shown in Fig.10. In fact the depth of the back face increases because of existence of crimp in the woven part of the panel as mentioned before. 


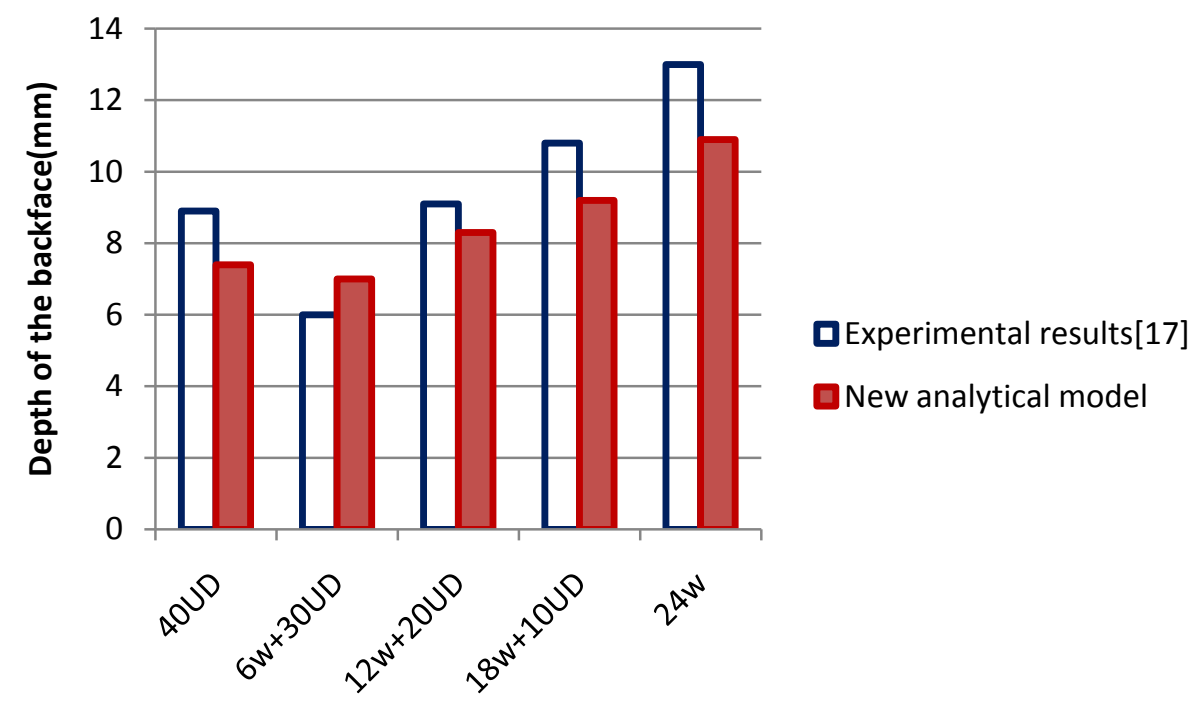

Fig. 10. Comparison of depth of the back face signature for 5 configurations of the panel.

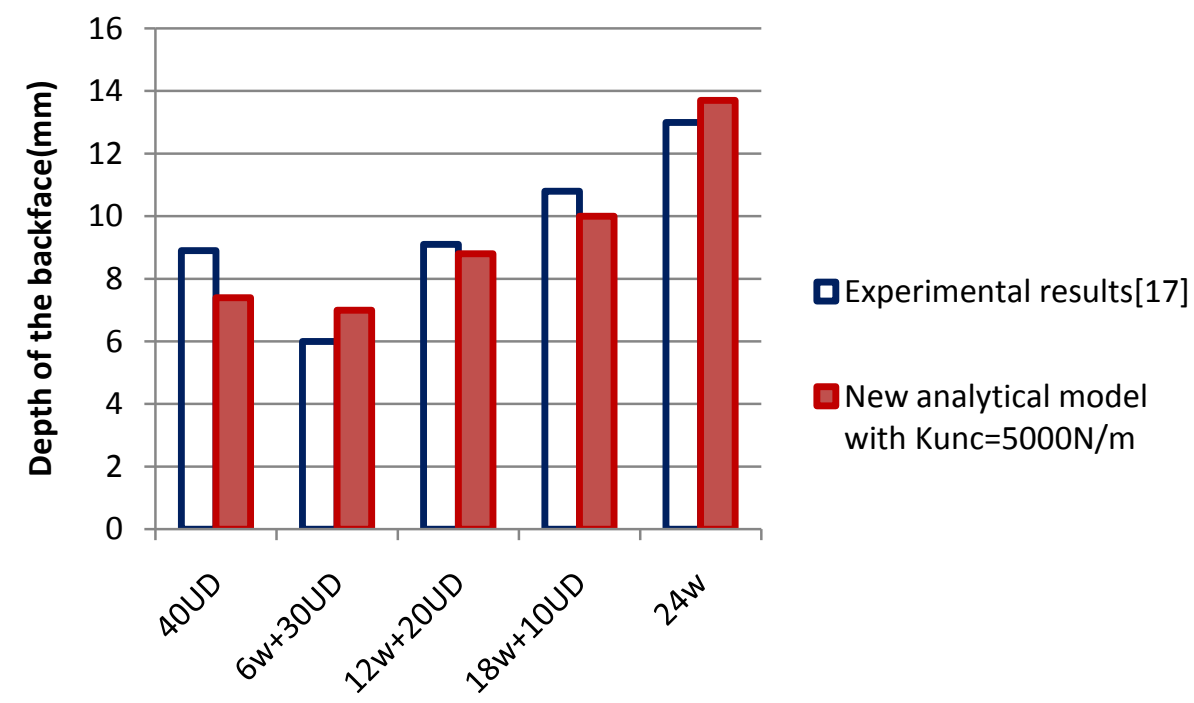

Fig. 11. Comparison of depth of the back face signature for 5 configurationsof the panel with considering Kunc $=5000 \mathrm{~N} / \mathrm{m}$.

\section{Conclusion}

A modified analytical model has been developed for hybrid woven/unidirectional panel subjected to ballistic impact. In this model a hybrid panel consists of woven fabric as front layer and unidirectional plies as back layers were analyzed. Crimp phenomena and reflection of transverse wave of intersection point in the fabric are considered in the model. Results show that the ballistic resistance of the hybrid woven/unidirectional panel is higher than panel with single material (woven fabric or unidirectional material). Also height of the pyramid of the back face signature in hybrid panel is less than pure woven fabrics panel with the same areal density. There is an optimum ratio of woven to UD materials in the hybrid ballistic panel depending on the material properties. 


\section{References}

1. M.Karbalaie, M.Yazdanirad, A.Mirhabibi: High performance Dyneema fiber laminate for impact resistance/macro structural composites. Journal of Thermoplastic Composite Materials 25(4), 403-411 (2011)

2. Park, J., Chi, Y.-S., Kang, T.: Ballistic performance of hybrid panels composed of unidirectional/woven fabrics. Textile Research Journa 83, 471 (2013)

3. Thomas, G.: Non-woven fabrics for military applications. In : M Tex WE (2008)

4. Karahan, M.: Comparsion of Ballistic Performance and energy absorbtion capabilities of woven and unidirectional aramid fabric. Tex Res J. 78, 718 (2008)

5. BZ (Gama) Haque and JW Gillespie, J.: A combined theoretical-semiempirical penetration model of ballistic penetration of thick section composites, Journal of Thermoplastic Composite Materials., vol.25 5: pp. (631-659) (2012)

6. K.Fin, B.: Performance Metric for composite integral armor, Journal of Thermoplastic Composite Materials., vol.13, 5, pp. 417-431 (2000)

7. Abatan, A., Hu, H.: Effect of cross section material distribution on impact response of hybrid composites. Journal of Thermoplastic Composite Materials 15, 375 (2002)

8. Liaghat, G., H.Shanazari, M.Aboutorabi, H.Hadavinia: A modified analytical model for analysis of perforation of projectile into ceramic composite targets. International J. Of Composite Materials $3(6 B), 17-22$ (2013)

9. Likavec, S.: On the impact characterization of polymer composite plates: dimensionless parameters and damage criteria. Journal of Thermoplastic Composite Materials 19, 575 (2006)

10. Smith, J., Frank, L.: Stress-strain relationship in yarn subjected to rapid impact loading: Part V: Wave propagation in long textile yarns impacted transversely. Tex J. Res 28, 288 (1958)

11. Jr., B.: A new penetration equation for ballistic limit analysis, Journal of Thermoplastic Composite Materials., vol.28 7: pp. 950-972 (2015)

12. Roylance, D.: Wave propagation in a viscoelastic fiber subjected to transverse impact. ASME J Appl Mech 40, 143-8 (1973)

13. Naik, N., Shrirao, P., Reddy, B.: Ballistic impact behavior of woven fabric composites: Formulation. Int. J. Impact Engineering 32, 1521-1552 (2006)

14. Mamivand, M., Liaghat, G.: A model for ballistic impact on multi-layer fabric targets. Int J Impact Eng 37(7), 806-12 (2010)

15. Ha-Minh, V., Imad, A., F.Boussu, F., Kanit, T.: On analytical modeling to predict of the ballistic 
impact behavior of textile multi-layer woven fabric. Composite Structures 99, 462-476 (2013)

16. Mohan, S., Velu, S.: Ballistic impact behavior of unidirectional fiber reinforced composites. Int. J. of impact engineering 63, 164-176 (2014)

17. Chen, X., Zhou, Y., Wells, G.: Numerical and experimental investigations into ballistic performance of hybrid fabric panels. Composites Part B 58, 35-42 (2014)

18. Chen, X., Zhu, F., Wells, G.: An analytical model for ballistic impact on textile based body armour. Composites Part B 45, 1508-1514 (2013)

19. Lim, J., Zheng, J., Masters, K., Chen, W.: Effect of gage length, loading rates, and damage on the PPTA fibres. Int J Impact Eng 38(4), 219-27 (2011)

20. Cheng, M., Chen, W., Weerasooriya, T.: Mechanical properties of Kevlar KM2 single fibre. J Eng Mater Technol ASME 127(2), 197-203 (2005)

21. Yildirim, K.: Weft directional stick-slip force of yarn pullout in para-aramid fabric for ballistics,Journal of Thermoplastic Composite Materials., vol.27, 9: pp. 1167-1191 (2014)

22. Kirkwood, K., Kirkwood, J., Lee, Y., Egres Jr., R.: Yarn Pull-Out as a Mechanism for Dissipation of Ballistic Impact Energy in Kevlar KM-2 Fabric, Part I: Quasi static Characterization of Yarn PullOut. (2004)

23. Liaghat, G., Abotorabi, M.: Thesis: Analytical and experimental investigation of penetration into Kevlar $^{\circledR}$ woven fabrics impregnated with a shear thickening fluid (STF)., Tehran

24. Rao, M., Duan, Y., Keefe, M., Powers, B., Bogetti, T.: Modeling the effects of yarn material properties and friction on the ballistic impact of a plain-weave fabric. Composite Structures 89, 556-566 (2009)

25. K. Bilisik, M.: multilayered and ultidirectional-stitched aramid Woven Fabric Structures Experimental Characterization of Ballistic Performance by Considering the Yarn Pull-out Test., vol.80(16): 1697-1720 (2012) 